\title{
Metal Artifact Reduction in Head CT Performed for Patients with Deep Brain Stimulation Devices: Effectiveness of a Single-Energy Metal Artifact Reduction Algorithm
}

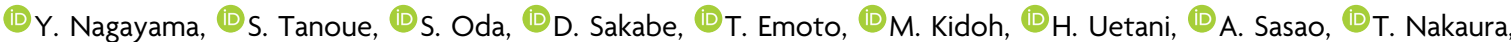

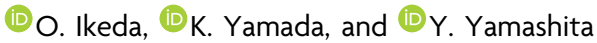

\begin{abstract}
BACKGROUND AND PURPOSE: Deep brain stimulation electrodes induce massive artifacts on CT images, deteriorating the diagnostic value of examinations. We aimed to investigate the usefulness and potential limitations of a single-energy metal artifact reduction algorithm in head CT performed in patients with implanted deep brain stimulation devices.
\end{abstract}

MATERIALS AND METHODS: Thirty-four patients with deep brain stimulation (bilateral, $n=28$ ) who underwent head CT on a 320 detector row scanner and whose images were reconstructed with and without single-energy metal artifact reduction at the examinations were retrospectively included. The severity of artifacts around electrodes was assessed objectively using SDs and an artifact index. Two radiologists subjectively evaluated the severity of artifacts from electrodes, the visibility of electrode localization and surrounding structures, and overall diagnostic confidence on 4-point scales. Background image quality (GM-WM contrast and image noise) was subjectively and objectively assessed. The presence and location of artifacts newly produced by single-energy metal artifact reduction were analyzed.

RESULTS: Single-energy metal artifact reduction provided lower objective and subjective metal artifacts and improved visualization of electrode localization and surrounding structures and diagnostic confidence compared with non-single-energy metal artifact reduction images, with statistical significance (all, $P<.01$ ). No significant differences were observed in GM-WM contrast and image noise (all, $P \geq$.11). The new artifacts from single-energy metal artifact reduction were prominently observed in patients with bilateral deep brain stimulation at high convexity, possibly induced by deep brain stimulation leads placed under the parietal scalp.

CONCLUSIONS: Single-energy metal artifact reduction substantially reduces the metal artifacts from deep brain stimulation electrodes and improves the visibility of intracranial structures without affecting background image quality. However, non-single-energy metal artifact reduction images should be simultaneously reviewed to accurately assess the entire intracranial area, particularly in patients with bilateral deep brain stimulation.

ABBREVIATIONS: DBS = deep brain stimulation; MAR = metal artifact reduction; SEMAR = single-energy metal artifact reduction

D eep brain stimulation (DBS) electrode implantation has been widely performed as an established surgical procedure for the treatment of various movement disorders such as Parkinson disease, medically intractable essential tremor, and dystonia. ${ }^{1}$ DBS has also been increasingly used for epilepsy, neuropathic pain, and psychiatric disorders. ${ }^{2}$ The main targets of

Received August 26, 2019; accepted after revision November 15.

From the Department of Diagnostic Radiology (Y.N., S.O., M.K., H.U., A.S., T.N., O.I., Y.Y.), Graduate School of Medical Sciences, and Departments of Neurosurgery (K.Y.) and Diagnostic Radiology (S.T.), Graduate School of Life Sciences, Kumamoto University, Kumamoto, Japan; and Department of Central Radiology (D.S., T.E.), Kumamoto University Hospital, Kumamoto, Japan.

Please address correspondence to Yasunori Nagayama, MD, PhD, Department of Diagnostic Radiology, Graduate School of Medical Sciences, Kumamoto University, 1-1-1, Honjo, Chuo-ku, Kumamoto 860-8556, Japan; e-mail: y.nagayama1980@gmail.com

http://dx.doi.org/10.3174/ajnr.A6375
DBS are the subthalamic nucleus, globus pallidus pars interna, and ventral intermediate nucleus of the thalamus. ${ }^{1,2} \mathrm{CT}$ is fast, easily accessible, and less expensive compared with MR imaging and is often used for evaluating the contact location of electrodes, surgical complications, and other intracranial abnormalities not related to a surgical procedure in patients implanted with DBS devices. However, metal artifacts induced by the electrodes usually hamper the visualization of implants and the surrounding intracranial structures, thereby increasing the potential risk of missing relevant findings and deteriorating the diagnostic utility of the examination. ${ }^{3}$

Metal artifacts are mainly introduced by 2 different physical phenomena: photon starvation as a consequence of a complete $\mathrm{x}$ ray photon absorption and beam-hardening caused by the preferential absorption of low-energy photons. ${ }^{4}$ The degree of $\mathrm{x}$-ray photon absorption and the resulting physical effects on CT 
Table 1: Patient and device characteristics

\begin{tabular}{lc}
\hline & Mean \pm SD or No. (\%) \\
\hline Age (yr) & $68.0 \pm 10.3$ \\
Male/female ratio & $10(29.4): 24(70.6)$ \\
Underlying disease & \\
$\quad$ Parkinson disease & $21(61.8)$ \\
Essential tremor & $6(17.6)$ \\
Dyskinesia & $5(14.7)$ \\
Others & $2(5.9)$ \\
Unilateral/bilateral DBS & $6(17.6) / 28(82.4)$ \\
DBS lead model & \\
Model 3387/3389 (Medtronic) & $53(85.5) / 9(14.5)$ \\
Targets of DBS & \\
STN/GPi/Vim & $42(67.7) / 11(17.7) / 9(14.5)$ \\
\hline
\end{tabular}

Note:-GPi indicates globus pallidus internal; STN, subthalamic nucleus; Vim, ventral intermediate nucleus of thalamus.

images depend on the composition, size, and the shape of the metallic hardware. $^{5-7}$ Small implants made of metal having a relatively low atomic number, such as a surgical clip made of titanium (atomic number 22), may cause minor beam-hardening. ${ }^{6-8}$ In contrast, hardware made of metals having a high atomic number, such as DBS electrodes made of platinum (atomic number 78) and iridium (atomic number 77), usually cause severe bright and dark streak artifacts due to photon starvation, deteriorating the image quality and diagnostic values of the CT examination. ${ }^{6,7}$

Several techniques, including increased tube voltage, tube current, and application of high kiloelectron volt virtual monoenergetic imaging from dual-energy CT, can aid in the reduction of metal artifacts. ${ }^{6,7}$ However, these approaches have limited value for handling photon starvation induced by large or high atomic number metallic implants. ${ }^{6,9,10}$ As an alternative approach, a single-energy metal artifact reduction (SEMAR) algorithm, a raw data-based metal artifact reduction (MAR) algorithm used in conventional single-energy CT, has been recently developed. ${ }^{11}$ This algorithm removes severe metal artifacts induced by photon starvation using multiple steps of data segmentation and interpolation along with repeat forward and back projections in the projection and image data domains. Thus far, a few studies have shown that SEMAR can substantially improve the image quality by reducing the metal artifacts in several clinical conditions. ${ }^{12-15}$ However, no prior investigations have evaluated the added value and possible limitations of using the SEMAR algorithm for head CT performed on the subgroup of patients implanted with DBS devices, in which strong metal artifacts have been observed.

This study aimed to investigate the impact of the SEMAR algorithm on the subjective and objective image quality of head CT performed in patients implanted with DBS devices.

\section{MATERIALS AND METHODS}

This retrospective study was approved by the institutional review board (Kumamoto University), and the requirement for written informed consent was waived.

\section{Patients}

Using our radiology data base, we retrospectively screened patients for inclusion among those implanted with DBS devices who underwent noncontrast head CT between January 2016 and December 2018. Our inclusion criteria were the following: 1) older than 18 years of age; 2 ) use of a standardized image-acquisition protocol on a 320-detector row multidetector CT system, as detailed later; and 3) absence of other metallic materials possibly affecting the artifact analysis. In total, 34 patients (10 men, 24 women; mean age, $68.0 \pm 10.3$ years) who fulfilled the above criteria were analyzed. Clinical indications for head CT were the evaluation of contact locations and surgical complications $(n=24)$; suspicion of cerebrovascular disease unlikely related to surgical procedures $(n=7)$; head trauma $(n=1)$; and follow-up of known cavernous hemangioma $(n=1)$ or pituitary tumor $(n=1)$. Among the included patients, 28 were implanted with bilateral DBS devices and 6 were implanted with unilateral DBS devices; thus, a total of 62 DBS electrodes were evaluated. Detailed patient demographics are summarized in Table 1.

\section{DBS Implantation Procedure}

All patients underwent a standard DBS implantation procedure in accordance with our institution's good clinical practice. In brief, DBS leads (Model-3387 for subthalamic nucleus-DBS and globus pallidus pars interna-DBS or Model-3389 for the ventral intermediate nucleus of the thalamus-DBS, both 40cm long: Medtronic; Minneapolis, Minnesota), composed of iridium alloy and containing 4 platinum-iridium electrodes near the tip, were placed within the target positions through burr-holes in the skull. After the operator ensured that the electrodes were correctly positioned to control movement symptoms, the excess extracranial DBS leads were looped under the scalp around the burr-holes. The extracranial DBS lead and internal pulse generator subcutaneously implanted in the subclavian portion of the chest were connected with the extension wire running under the skin from the chest to the neck and head (Fig 1, left).

\section{Image Acquisition}

A 320-detector row multidetector CT system (Aquilion ONE, VISION Edition; Canon Medical Systems, Otawara, Japan) was used for scanning. Patients were scanned in the supine position with the following parameters: tube voltage, $120 \mathrm{kV}$ (peak); tube current, $260 \mathrm{~mA}$ (without automated modulation); rotation time, 1.0 seconds; pitch factor, 0.637 ; detector collimation, $80 \times 0.5 \mathrm{~mm}$. For each patient, 2 sets of axial images with $5-\mathrm{mm}$ slices were reconstructed: one using the iterative reconstruction technique (adaptive iterative dose reconstruction [AIDR] 3D; Canon Medical Systems) at the noise-reduction level of "weak," referred to as nonSEMAR images, and the second using the AIDR 3D (weak) plus the SEMAR algorithm, referred to as SEMAR images. Both image series were reconstructed when clinical CT was performed as part of the original head CT examination.

\section{Quantitative Image Analysis}

Quantitative image analyses were performed in consensus by 2 board-certified radiologists having 11 and 14 years of experience in neuroimaging. In this session, all images were reviewed together by both the radiologists. At the section level exhibiting visually the most pronounced artifacts from electrodes, 4 circular 


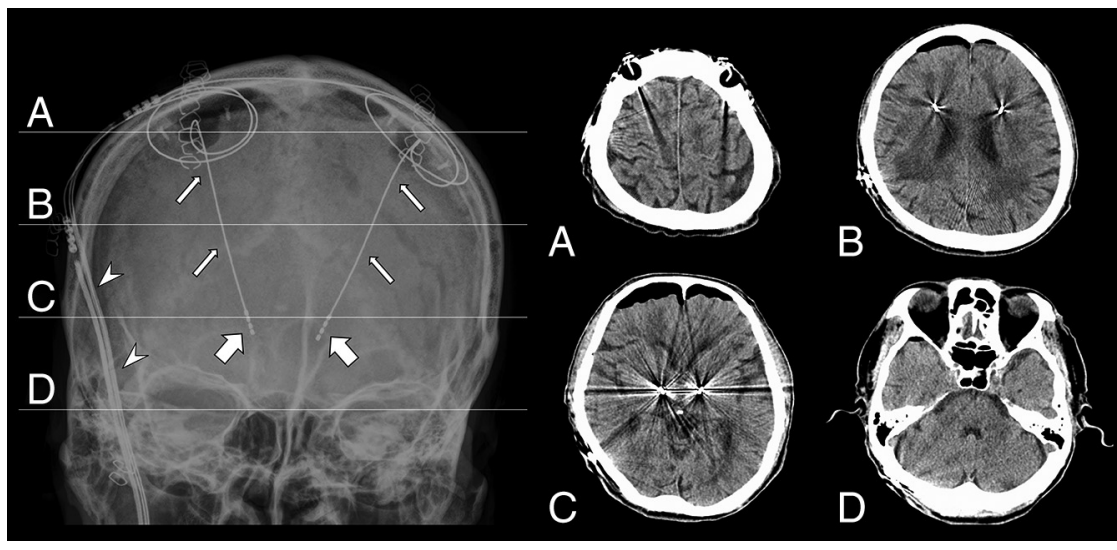

FIG 1. Left, postoperative radiographic image of a 67-year-old man implanted with a bilateral subthalamic nucleus-DBS lead. The DBS system comprises leads (small arrows), electrodes located at lead tips (large arrows), and subcutaneous extension cables (arrowheads) connecting leads to the pulse generator subcutaneously implanted in the right chest wall (not shown). The excess extracranial part of DBS leads was looped under the parietal scalp. The horizontal lines show the section levels of high convexity $(A)$, corona radiata $(B)$, basal ganglia $(C)$, and posterior fossa (D). Right, axial non-SEMAR CT images at the section levels corresponding to the lines drawn in the left radiographic image $(A-D)$, where the presence and degree of artifacts created by SEMAR reconstruction were separately scored.

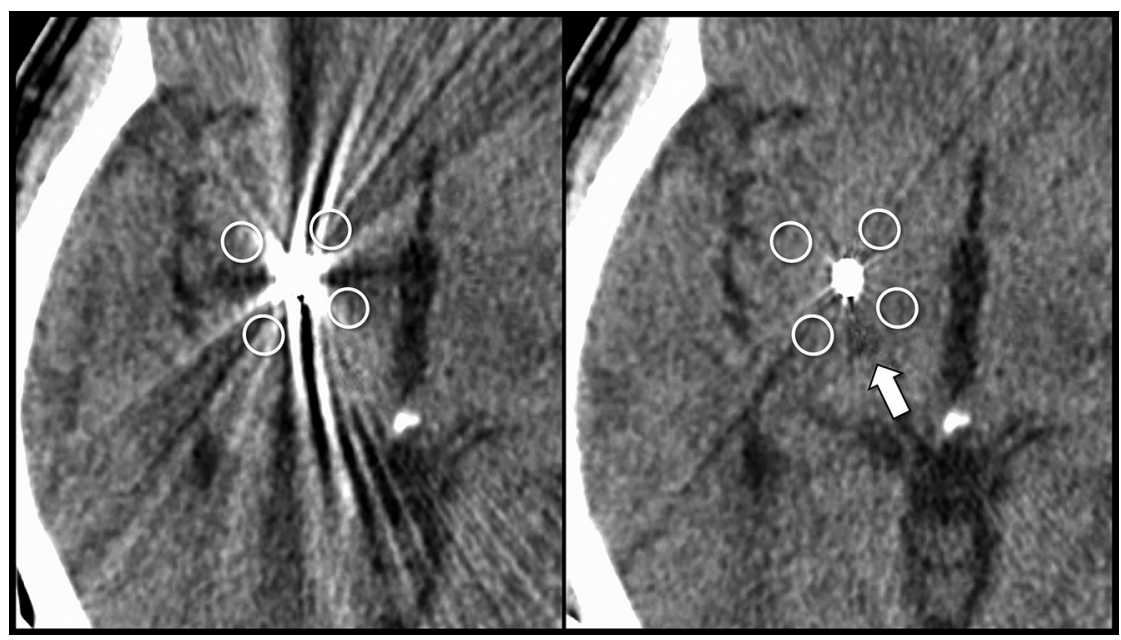

FIG 2. Axial postoperative CT images of a 55-year-old man with dystonia secondary to right thalamic hemorrhage treated by unilateral globus pallidus pars interna-DBS. To quantify the artifact severity, we placed 4 ROIs around the electrodes on non-SEMAR (left) and SEMAR (right) images. The ROI setting was consistent between both images. Compared with non-SEMAR images, SEMAR images substantially reduce artifacts from electrodes and clearly depict the old hemorrhagic changes in the right thalamus (arrow).

ROIs of approximately $20 \mathrm{~mm}^{2}$ were drawn around each electrode (Fig 2). ${ }^{13}$ Additionally, ROIs were placed on the cerebral white matter (approximately $50 \mathrm{~mm}^{2}$ ) and adjacent cortical gray matter (approximately $10 \mathrm{~mm}^{2}$ ) on the frontal lobe at the section level not or least affected by the metal artifacts, ${ }^{10}$ while the visible artifacts, focal lesions, and adjacent structures were excluded from the ROIs. The mean and SD of the CT attenuation in each ROI were recorded. The averaged SD of the 4 ROIs placed around each DBS electrode was considered an index of the metal artifacts. ${ }^{13}$ For minimizing the effect of background image noise on the metal artifact quantification, we also calculated the artifact index (AI) as follows: ${ }^{12,16}$
$\mathrm{AI}=\sqrt{ }[(\mathrm{SD}$ around the $\mathrm{DBS}$

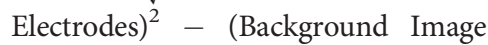
Noise $)^{2}$.

In this study, the SD at the cerebral WM on the frontal lobe was defined as the background image noise in accordance with the literature. ${ }^{10}$ The GM-WM contrast was calculated as the difference between the CT attenuations of the GM and WM. This value was divided by the background image noise to calculate the contrast-to-noise ratio. The GM-WM contrast and contrast-to-noise ratio were not measured for the area affected by artifacts because the presence of dark and bright artifacts could make the CT attenuation unreliable for representing brain tissue contrast. The size and location of each ROI were kept constant for non-SEMAR and SEMAR images using the copy and paste function, and quantifications were performed twice to retain data consistency using the averaged values for the analysis. The mean and SD of each quantitative value among 34 patients were calculated for non-SEMAR and SEMAR images.

\section{Qualitative Image Analysis}

Two board-certified radiologists having 8 and 11 years of experience who were blinded to the reconstruction algorithms and patient information independently evaluated the nonSEMAR and SEMAR image quality in random order using 4-point scales. Scoring was performed on images without any features to help readers identify the SEMAR and non-SEMAR images. The window level and width were set at 35 and $80 \mathrm{HU}$, respectively. At the section levels with the most severe artifacts, the severity of metal artifacts from DBS electrodes was assessed as $1=$ severe (unacceptable); 2 = moderate (acceptable only under limited conditions); $3=$ mild (mostly acceptable); $4=$ minimal/absent. The visibility of the electrode localization and intracranial anatomic landmarks (basal ganglia, thalamus, cortico-medullary junction, lateral ventricle, third ventricle, and Sylvian fissure) were scored as $1=$ no visualization; $2=$ poor (only partially visible); $3=$ moderate (mostly visible); and $4=\operatorname{good}$ (completely visible). At the region not or minimally affected by the artifacts, the background image quality was assessed on the basis of the image noise and GM-WM contrast $(1=$ undiagnostic; $2=$ suboptimal; 3 = average; and $4=$ excellent). Then, the overall 
diagnostic confidence was graded as $1=$ undiagnostic and 2 = diagnostic only under limited conditions; $3=$ mostly diagnostic; and $4=$ completely diagnostic. Interreader disagreement was resolved by consensus during a subsequent joint reading to determine the final score. The mean and SD of each score obtained from 34 patients were calculated for SEMAR and non-SEMAR images.

\section{Assessment of New Artifacts Induced by SEMAR}

Subsequent to the above blinded qualitative assessments, readers were asked to simultaneously review the SEMAR and nonSEMAR images and to determine the presence and degree of new artifacts developed by the SEMAR algorithm on a 4-point scale $(1=$ severe, unacceptable; $2=$ moderate, partially impaired diagnostic confidence; 3 = mild, mostly acceptable; and 4 = absent). In this session, readers were informed about which image was reconstructed with or without SEMAR. The scoring was performed separately for each section level of high convexity, corona radiata, basal ganglia, and posterior fossa. Figure 1 shows a typical cranial radiographic image of patients implanted with bilateral DBS devices (left) and corresponding axial non-SEMAR images at the section levels for the evaluation of a new artifacts (right). Interreader disagreement was resolved by consensus during a joint reading to determine the final score. The mean and SD values of each final score among 34 patients were calculated for SEMAR and non-SEMAR images.

Table 2: Quantitative analysis ${ }^{a}$

\begin{tabular}{lccc}
\hline & Non-SEMAR & SEMAR & $\begin{array}{c}\boldsymbol{P} \\
\text { Value }\end{array}$ \\
\hline $\begin{array}{l}\text { Severity of metallic artifacts } \\
\text { SD around DBS electrodes } \\
\text { (HU) }\end{array}$ & $28.3 \pm 9.1$ & $9.8 \pm 3.2$ & $<.01$ \\
Artifact index (HU) & $28.0 \pm 9.2$ & $9.0 \pm 3.5$ & $<.01$ \\
Background image quality & & & \\
GM-WM contrast (HU) & $11.7 \pm 2.7$ & $11.6 \pm 2.4$ & .35 \\
Image noise (HU) & $3.8 \pm 0.7$ & $3.7 \pm 0.7$ & .17 \\
CNR & $3.2 \pm 1.0$ & $3.2 \pm 0.8$ & .65 \\
\hline
\end{tabular}

Note:-CNR indicates contrast-to-noise ratio; HU, Housfield unit.

${ }^{a}$ Data are means.

\section{Statistical Analysis}

Statistical analyses were performed using a statistical software package ( $\mathrm{R}$ statistical and computing software, Version 3.2.2; www.r-project.org/). All numeric values are expressed as mean \pm $\mathrm{SD}$, whereas categoric variables are expressed as proportions (percentage). The quantitative measurements between the SEMAR and non-SEMAR images were compared using the paired $t$ test after the normality of the distribution was confirmed using the Kolmogorov-Smirnov test. The subjective scores were compared using the Wilcoxon signed rank test. The interobserver agreement was assessed with the $\kappa$ values $(\leq 0.20=$ poor, $0.21-$ $0.40=$ fair, $0.41-0.60=$ moderate, $0.61-0.80=$ substantial, and $0.81-1.00=$ perfect $) . P<.05$ was considered significant.

\section{RESULTS}

\section{Quantitative Analysis}

Table 2 summarizes the results of quantitative image analysis. The mean and SD around DBS electrodes and artifact index values for SEMAR images were significantly lower than those for non-SEMAR images $(P<.01)$. For the brain segments not affected by metal artifacts, no significant differences in GM-WM contrast, image noise, and contrast-to-noise ratio were observed between both images $(P=.17-.65)$.

\section{Qualitative Analysis}

Table 3 shows the qualitative image-analysis results. SEMAR images provided significantly fewer metal artifacts and improved visibility of electrode localization and surrounding anatomic structures compared with non-SEMAR images $(P<.01)$. Subjective scores for GM-WM contrast $(P=.11)$ and image noise $(P=.32)$ at the region not affected by metal artifacts were not significantly different between SEMAR and non-SEMAR images. The overall diagnostic confidence was significantly higher in SEMAR compared with non-SEMAR images $(P<.01)$. Interobserver agreement was moderate to substantial $(\kappa=0.51-0.72)$ for all evaluation criteria. Before the consensus reading, the score disagreement between readers in the visibility of structures, background of image quality, and overall diagnostic confidence ranged from $2 / 34$ to $8 / 34$ cases (5.9\%-23.5\%) and $1 / 34$ to $9 / 34$ cases $(2.9 \%-26.5 \%)$ of non-SEMAR and SEMAR image series, respectively.

Table 3: Qualitative image analysis ${ }^{\mathrm{a}}$

\begin{tabular}{|c|c|c|c|c|c|}
\hline & \multirow[b]{2}{*}{ Non-SEMAR } & \multirow[b]{2}{*}{ SEMAR } & \multirow[b]{2}{*}{$P$ Value } & \multicolumn{2}{|c|}{$\kappa$ Value } \\
\hline & & & & Non-SEMAR & SEMAR \\
\hline The severity of metallic artifacts & $1.2 \pm 0.4$ & $2.9 \pm 0.2$ & $<.01$ & $0.53(5)$ & $0.65(1)$ \\
\hline \multicolumn{6}{|l|}{ Visibility of structures } \\
\hline Electrode localization & $1.5 \pm 0.6$ & $3.5 \pm 0.5$ & $<.01$ & $0.62(8)$ & $0.53(8)$ \\
\hline Basal ganglia & $1.2 \pm 0.4$ & $3.0 \pm 0.4$ & $<.01$ & $0.57(5)$ & $0.61(5)$ \\
\hline Thalamus & $1.1 \pm 0.3$ & $2.8 \pm 0.5$ & $<.01$ & $0.63(2)$ & $0.66(5)$ \\
\hline Cortico-medullary junction & $1.8 \pm 0.3$ & $3.4 \pm 0.5$ & $<.01$ & $0.51(7)$ & $0.72(5)$ \\
\hline Sylvian fissure & $2.1 \pm 0.7$ & $3.7 \pm 0.5$ & $<.01$ & $0.58(8)$ & $0.58(6)$ \\
\hline Lateral ventricle & $2.0 \pm 0.5$ & $3.8 \pm 0.4$ & $<.01$ & $0.70(4)$ & $0.53(9)$ \\
\hline Third ventricle & $1.6 \pm 0.5$ & $3.2 \pm 0.7$ & $<.01$ & $0.56(7)$ & $0.58(6)$ \\
\hline \multicolumn{6}{|l|}{ Background image quality } \\
\hline GM-WM contrast & $3.2 \pm 0.6$ & $3.3 \pm 0.6$ & .11 & $0.60(8)$ & $0.68(7)$ \\
\hline Image noise & $3.2 \pm 0.4$ & $3.2 \pm 0.4$ & .32 & $0.61(4)$ & $0.53(4)$ \\
\hline Overall diagnostic confidence & $1.4 \pm 0.5$ & $3.1 \pm 0.4$ & $<.01$ & $0.52(8)$ & $0.56(4)$ \\
\hline
\end{tabular}

${ }^{a}$ Data are means. Parentheses show the number of cases for which the subjective score before consensus reading was disputed by both readers. Scale for subjective score: 1 = undiagnostic, 2 = suboptimal, 3 = acceptable, 4 = fully diagnostic. 


\begin{tabular}{lcccc}
\hline & & \multicolumn{2}{c}{ Subgroup Analysis } \\
\cline { 3 - 5 } Section Levels & All Patients & Unilateral DBS & Bilateral DBS & $P$ Value \\
\hline High convexity & $2.7 \pm 0.8$ & $3.7 \pm 0.5$ & $2.5 \pm 0.6$ & $3.9 \pm 0.4$ \\
Corona radiata & $3.9 \pm 0.3$ & $4.0 \pm 0$ & $3.9 \pm 0.3$ & .30 \\
Basal ganglia & $3.9 \pm 0.2$ & $4.0 \pm 0$ & $3.7 \pm 0.6$ & .49 \\
Posterior fossa & $3.7 \pm 0.6$ & $4.0 \pm 0$ & .15 \\
\hline
\end{tabular}

${ }^{a}$ Data are means. Scale for subjective score: $1=$ severe, $2=$ moderate, $3=$ mild, 4 = absent.

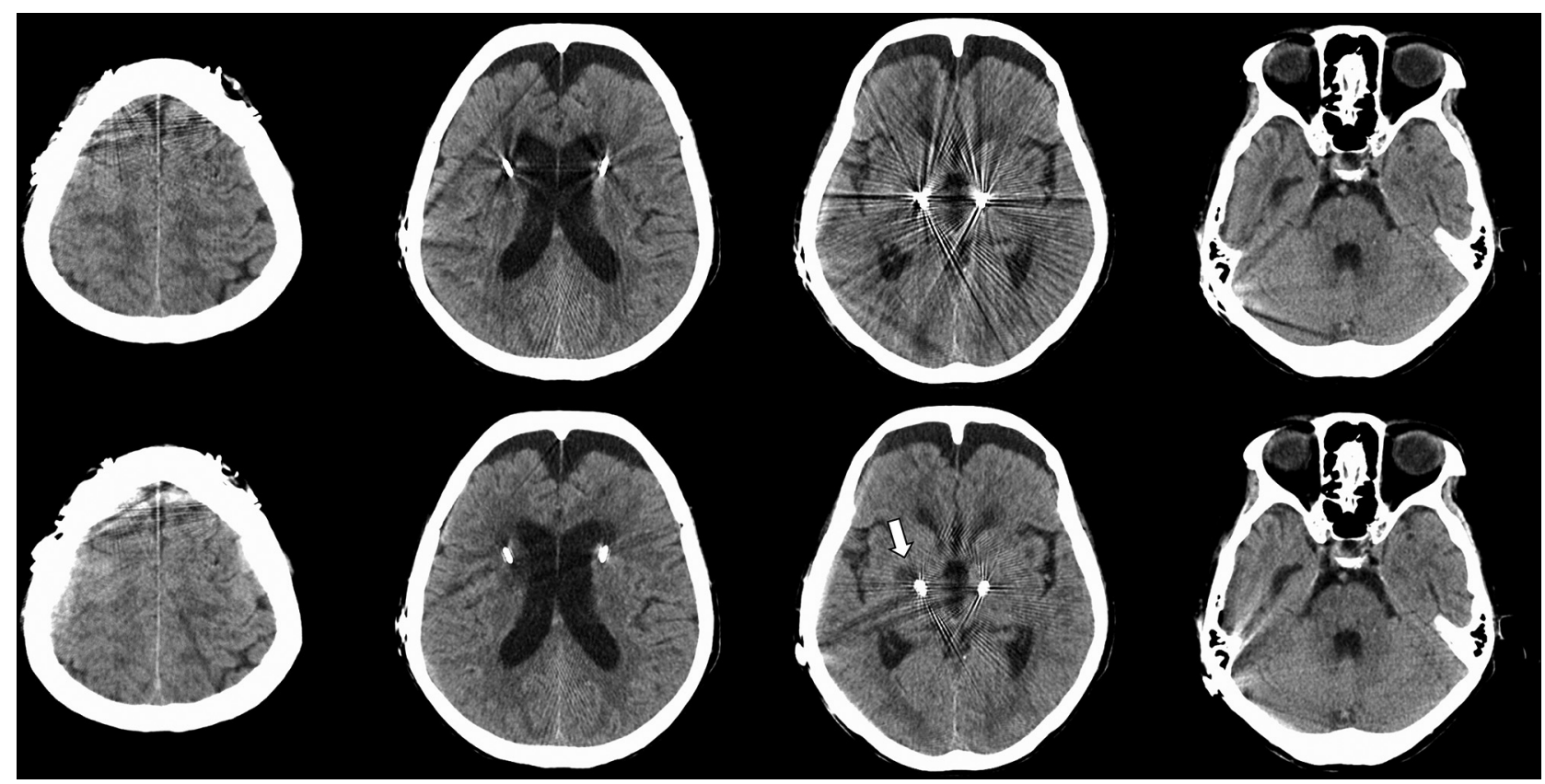

FIG 3. Axial CT images of a 71-year-old woman with Parkinson disease treated with bilateral subthalamic nucleus-DBS. Compared with nonSEMAR images (upper row), SEMAR images (lower row) substantially reduce artifacts from intracranial leads and electrodes, without compromising the brain tissue contrast. Note that the low-attenuation area of old cerebral hemorrhage around the right DBS lead is clearly visible only in SEMAR images. However, new artifacts possibly induced by looped DBS leads under the parietal scalp are observed in the SEMAR image at the section level of high convexity.

\section{New Artifacts Induced by the SEMAR Algorithm}

During simultaneous comparisons, new artifacts created by SEMAR, such as smudged fuzzy high-attenuation areas were prominently observed at the section level of higher convexity in patients implanted with bilateral DBS devices, whereas they were scantly observed at other section levels and in patients implanted with unilateral DBS devices (Table 4). These new artifacts were mainly derived from looped DBS leads placed under the parietal scalp, degrading the depiction of some intracranial structures such as the sulcus and cortico-medullary junction that could be more clearly visualized in non-SEMAR images (Fig 3). Interobserver agreement for assessing new artifacts was moderate to substantial $(\kappa=0.52-0.67)$.

\section{DISCUSSION}

This study demonstrates that SEMAR could improve the visibility of numerous intracranial anatomic landmarks and the diagnostic confidence of head CT for patients with DBS by reducing the metal artifacts from electrodes. Additionally, we confirmed that subjective and objective background image quality (ie, GM-WM contrast and image noise) was identical between SEMAR and non-SEMAR images. In contrast, despite the substantial improvement in the overall diagnostic confidence, the SEMAR algorithm was found to potentially introduce new artifacts, particularly at the area with higher convexity in patients with bilateral DBS.

The usefulness of SEMAR has been noted for patients with several types of metallic implants, ${ }^{12-15,17}$ while only 2 studies have addressed its feasibility for cranial CT. ${ }^{14,15}$ Pan et al ${ }^{14}$ demonstrated that SEMAR could reduce the artifacts from neurosurgical clips or endovascular coils without affecting the quantitative perfusion parameters compared with non-SEMAR images. Katsura et $\mathrm{al}^{15}$ also revealed that SEMAR substantially reduced the artifacts from platinum endovascular coils while preserving the arterial iodine contrast by analyzing 20 patients who underwent postinterventional cerebral CT angiography. Despite these suggestive findings, no prior study has applied the SEMAR for noncontrast head CT performed for patients with DBS. Given that the physical effects causing metal artifacts are primarily dependent on the geometry and composition of the metallic devices, ${ }^{5-7}$ the effects of MAR algorithms on the image quality should be analyzed for individual clinical situations. In this context, our study 
revealed that SEMAR could be an effective and suitable technique for evaluating intracranial abnormalities around the DBS electrodes, providing the evidence for an additional clinical applicability of this algorithm. As shown in Figs 2 and 3, some findings hidden under severe streaks in non-SEMAR images could be clearly depicted after SEMAR application, possibly yielding improved diagnostic values and optimal patient management while eliminating the necessity for additional MR imaging.

The reconstruction of higher kiloelectron volt virtual monoenergetic imaging from dual-energy CT is another established technique to reduce metal artifacts. ${ }^{6,7}$ This approach is beneficial for suppressing beam-hardening induced by small and low atomic number metals but requires specific dual-energy CT scanners that are less available than the conventional single-energy CT and may be insufficient for removing bright and dark streaks caused by photon starvation from metals with a large mass or higher atomic number such as DBS electrodes. ${ }^{6,9,10,17,18}$ Furthermore, increasing the $\mathrm{x}$-ray energy decreases the soft-tissue contrast (eg, GM-WM contrast) in head CT images due to the reduced photoelectric effect. ${ }^{10,19-22}$ Such a deterioration of lowcontrast resolution may obscure relevant anatomic structures and certain intracranial abnormalities. ${ }^{21}$ In contrast, the SEMAR algorithm was primarily developed to mitigate the photon starvation induced by metals on conventional single-energy CT, and it only works on pixels segmented as metal on the original image data based on the attenuation threshold. In line with a few reports showing that the iodine contrast enhancement could be preserved in SEMAR images, ${ }^{15,17}$ we also confirmed that SEMAR could maintain the more subtle attenuation difference of GM-WM contrast (approximately $10 \mathrm{HU}$ ) while drastically reducing metal artifacts. The other important advantage of SEMAR relative to the dual-energy CT technique is that artifact reduction can be retrospectively achieved without prospective patient selection, optimizing scan parameters before image acquisition and increasing the radiation dose.

Other than SEMAR, dedicated MAR algorithms have been developed and investigated for patients with DBS. ${ }^{10,23}$ Aissa et $\mathrm{al}^{23}$ demonstrated that Iterative Metal Artifact Reduction (iMAR; Siemens, Erlangen, Germany) could reduce metal artifacts near DBS electrodes compared with the filtered back-projection algorithm by analyzing 17 patients who underwent head CT after DBS implantation. Furthermore, Große Hokamp et $\mathrm{al}^{10}$ revealed that O-MAR (Philips Healthcare, Best, the Netherlands) could effectively reduce the artifacts from DBS electrodes compared with the conventional iterative reconstruction or high-kiloelectron volt virtual monoenergetic imaging. Our results were mostly concordant with these preliminary findings despite the substantial differences in imaging properties of each MAR algorithm. 9,18,24,25

Previous studies have demonstrated that "new artifacts" could be generated around metallic devices by applying MAR algorithms. ${ }^{5,9,18,24}$ However, their effect on the image quality has not been assessed in SEMAR images in patients implanted with DBS devices. $^{10,23}$ New artifacts from SEMAR were prominently observed in the slices with high convexity levels in patients with bilateral DBS, whereas they were scantily observed at the other section levels and in patients with unilateral DBS. The potential explanation of this observation is that the image data at high convexity include multiple thin metal fragments (ie, looped excess DBS leads placed under the parietal scalp) in-plane. ${ }^{26}$ Because SEMAR uses a Hounsfield unit threshold for metal segmentation, these conditions might cause a missed metal segmentation and inaccurate interpolation due to metal-induced scattering, partial volume, and beam-hardening artifacts, particularly in patients with bilateral DBS, thus, introducing new artifacts in final SEMAR images. ${ }^{5,6}$ Although these artifacts could affect only limited anatomic areas and overall diagnostic confidence was substantially improved on SEMAR images, non-SEMAR images should be simultaneously reviewed to avoid misinterpretation.

The past several years have seen an increase in the use of artificial intelligence, such as deep learning in medical imaging, and its feasibility has been exploited for mitigating CT metal artifacts. ${ }^{27,28}$ For instance, convolutional neural networks have been applied to correct the sinogram data corrupted by metals. Preliminary results have suggested that such a deep learning-based MAR approach outperforms existing MAR algorithms in terms of metal artifact suppression and preservation of the boundary between the implanted metal and surrounding tissue, which is usually difficult to correctly recover in commercially available MAR. ${ }^{27,28}$ Validating deep learning based MAR algorithms clinical utility and comparison with existing MAR algorithms for DBS settings may be interesting research subjects in the future.

This study has several limitations. First, this is a retrospective single-center study that includes a relatively small number of patients with DBS. Although our sample size was larger than that of recent studies focusing on MAR in head CT, ${ }^{10,15,23}$ further studies are required to confirm our results. Second, we could not compare the diagnostic performance of SEMAR and non-SEMAR images because of the lack of a sufficient reference standard in most cases. We focused on evaluating subjective and objective image quality to reveal the potential values and limitations of SEMAR as a standard-of-care head CT for DBS settings. Further investigations should be performed to clarify whether the additive use of SEMAR could improve the diagnostic accuracy, patient management, and clinical consequences. Third, in qualitative analysis, the interrater agreement for some variables was relatively limited, possibly due to the inherent nature of subjective assessment. Nevertheless, subsequently performed consensus reading could mitigate the individual assessment bias and make our results generalizable. Last, our results were limited to images obtained with vendorspecific algorithms and our standard-of-care 320-detector row CT protocol and may not be directly transposable to images obtained with other MAR algorithms and different acquisition protocols. ${ }^{9,18,24}$ Because we could not directly compare the performance of SEMAR with that of different MAR techniques, further investigations are warranted to clarify the most effective MAR for handling the artifacts from DBS devices, particularly for institutions equipped with multiple CT scanners.

\section{CONCLUSIONS}

The application of the SEMAR algorithm in patients implanted with DBS devices substantially reduces metal artifacts from 
electrodes and improves visualization of numerous anatomic landmarks and the diagnostic confidence of head CT. Given that the new artifacts could be introduced by SEMAR, particularly at the high-convexity levels in patients with bilateral DBS, the combined review of SEMAR and non-SEMAR images might be recommended for more accurate image interpretation.

\section{REFERENCES}

1. Fasano A, Lozano AM. Deep brain stimulation for movement disorders: 2015 and beyond. Curr Opin Neurol 2015;28:423-36 CrossRef Medline

2. Holtzheimer PE, Mayberg HS. Deep brain stimulation for psychiatric disorders. Annu Rev Neurosci 2011;34:289-307 CrossRef Medline

3. Sitz A, Hoevels M, Hellerbach A, et al. Determining the orientation angle of directional leads for deep brain stimulation using computed tomography and digital $x$-ray imaging: a phantom study. Med Phys 2017;44:4463-73 CrossRef Medline

4. Barrett JF, Keat N. Artifacts in CT: recognition and avoidance. Radiographics 2004;24:1679-91 CrossRef Medline

5. Han SC, Chung YE, Lee YH, et al. Metal artifact reduction software used with abdominopelvic dual-energy CT of patients with metal hip prostheses: assessment of image quality and clinical feasibility. AJR Am J Roentgenol 2014;203:788-95 CrossRef Medline

6. Katsura M, Sato J, Akahane M, et al. Current and novel techniques for metal artifact reduction at CT: practical guide for radiologists. Radiographics 2018;38:450-61 CrossRef Medline

7. Gjesteby L, de Man BD, Jin Y, et al. Metal artifact reduction in CT: where are we after four decades? IEEE Access 2016;4:5826-49. https://ieeexplore.ieee.org/document/7565564. Accessed April 3, 2019

8. Nagayama Y, Nakaura T, Tsuji A, et al. Cerebral bone subtraction CT angiography using $80 \mathrm{kVp}$ and sinogram-affirmed iterative reconstruction: contrast medium and radiation dose reduction with improvement of image quality. Neuroradiology 2017;59:127-34 CrossRef Medline

9. Andersson KM, Norrman E, Geijer H, et al. Visual grading evaluation of commercially available metal artefact reduction techniques in hip prosthesis computed tomography. Br J Radiol 2016;89:20150993 CrossRef Medline

10. Große Hokamp N, Hellerbach A, Gierich A, et al. Reduction of artifacts caused by deep brain stimulating electrodes in cranial computed tomography imaging by means of virtual monoenergetic images, metal artifact reduction algorithms, and their combination. Invest Radiol 2018;53:424-31 CrossRef Medline

11. Chang YB, Xu D, Zamyatin AA. Metal artifact reduction algorithm for single energy and dual-energy CT scans. In: Proceedings of the 2012 IEEE Nuclear Science Symposium and Medical Imaging Conference Record (NSS/MIC), Anaheim, California. October 27 to November 3, 2012:3426-29

12. Yasaka $\mathrm{K}$, Kamiya $\mathrm{K}$, Irie $\mathrm{R}$, et al. Metal artefact reduction for patients with metallic dental fillings in helical neck computed tomography: comparison of adaptive iterative dose reduction 3D (AIDR 3D), forward-projected model-based iterative reconstruction solution (FIRST) and AIDR 3D with single-energy metal artefact reduction (SEMAR). Dentomaxillofac Radiol 2016;45:20160114 CrossRef Medline

13. Kidoh M, Utsunomiya D, Ikeda O, et al. Reduction of metallic coil artefacts in computed tomography body imaging: effects of a new single-energy metal artefact reduction algorithm. Eur Radiol 2016;26:1378-86 CrossRef Medline
14. Pan YN, Chen G, Li AJ, et al. Reduction of metallic artifacts of the post-treatment intracranial aneurysms: effects of single energy metal artifact reduction algorithm. Clin Neuroradiol 2019;29:27784 CrossRef

15. Katsura M, Sato J, Akahane M, et al. Single-energy metal artifact reduction technique for reducing metallic coil artifacts on postinterventional cerebral CT and CT angiography. Neuroradiology 2018;60:1141-50 CrossRef Medline

16. Dong $\mathrm{Y}$, Shi $\mathrm{AJ}, \mathrm{Wu} \mathrm{JL}$, et al. Metal artifact reduction using virtual monochromatic images for patients with pedicle screws implants on CT. Eur Spine J 2016;25:1754-63 CrossRef Medline

17. Kidoh $M$, Utsunomiya $D$, Oda $S$, et al. CT venography after knee replacement surgery: comparison of dual-energy CT-based monochromatic imaging and single-energy metal artifact reduction techniques on a 320-row CT scanner. Acta Radiol Open 2017;6:2058460117693463 CrossRef Medline

18. Andersson KM, Nowik P, Persliden J, et al. Metal artefact reduction in CT imaging of hip prostheses-an evaluation of commercial techniques provided by four vendors. Br J Radiol 2015;88:20140473 CrossRef Medline

19. Pomerantz SR, Kamalian S, Zhang D, et al. Virtual monochromatic reconstruction of dual-energy unenhanced head CT at 65-75 keV maximizes image quality compared with conventional polychromatic CT. Radiology 2013;266:318-25 CrossRef Medline

20. Zhao XM, Wang M, Wu RZ, et al. Dual-layer spectral detector CT monoenergetic reconstruction improves image quality of non-contrast cerebral CT as compared with conventional single energy CT. Eur J Radiol 2018;103:131-38 CrossRef Medline

21. Lennartz S, Laukamp KR, Neuhaus V, et al. Dual-layer detector CT of the head: initial experience in visualization of intracranial hemorrhage and hypodense brain lesions using virtual monoenergetic images. Eur J Radiol 2018;108:177-83 CrossRef Medline

22. Nagayama $Y$, Nakaura T, Tsuji A, et al. Radiation dose reduction using $100-\mathrm{kVp}$ and a sinogram-affirmed iterative reconstruction algorithm in adolescent head CT: impact on grey-white matter contrast and image noise. Eur Radiol 2017;27:2717-25 CrossRef Medline

23. Aissa J, Boos J, Schleich C, et al. Metal artifact reduction in computed tomography after deep brain stimulation electrode placement using iterative reconstructions. Invest Radiol 2017;52:18-22 CrossRef Medline

24. Bolstad K, Flatabo S, Aadnevik D, et al. Metal artifact reduction in CT, a phantom study: subjective and objective evaluation of four commercial metal artifact reduction algorithms when used on three different orthopedic metal implants. Acta Radiol 2018;59:1110-18 CrossRef Medline

25. Chou R, Li JH, Ying LK, et al. Quantitative assessment of three vendor's metal artifact reduction techniques for $\mathrm{CT}$ imaging using a customized phantom. Comput Assist Surg (Abingdon) 2019;24:34-42 CrossRef Medline

26. Golestanirad L, Kirsch J, Bonmassar G, et al. RF-induced heating in tissue near bilateral DBS implants during MRI at 1.5T and 3T: the role of surgical lead management. Neuroimage 2019;184:566-76 CrossRef Medline

27. Ghani MU, Karl WC. Deep learning based sinogram correction for metal artifact reduction. Electronic Imaging 2018;2018:472128 CrossRef

28. Zhang Y, Yu H. Convolutional neural network-based metal artifact reduction in $\mathbf{x}$-ray computed tomography. IEEE Trans Med Imaging 2018;37:1370-81 CrossRef Medline 